\title{
Glucose Uptake During Exercise in Skeletal Muscles Evaluated by Positron Emission Tomography
}

\author{
Hiroyuki Shimada \\ Section for Health Promotion, Department of Health and Medical Care, \\ Center for Development of Advanced Medicine for Dementia, \\ National Center for Geriatrics and Gerontology \\ Japan
}

\section{Introduction}

Traditionally, in vivo skeletal muscle function has been investigated with noninvasive techniques such as magnetic resonance (MR) imaging that can characterize the motion and mechanics of contracting skeletal muscle (Axel \& Dougherty, 1989; Drace \& Pelc, 1994; Pipe et al., 1991). Other techniques include kinetic analyses to examine muscle activity during walking and electromyography (EMG) to evaluate muscle activity as amplitude-based algorithms. However, these techniques are limited because MR cannot be used to measure the metabolic activity of skeletal muscle, and kinetic analyses cannot measure isolated synergistic muscular activities or provide information on the etiology of the metabolic cost of exercise. Moreover, EMG quantification requires normalization of EMG amplitude to the EMG amplitude of maximal voluntary contractions, which some elderly are unable to achieve (Stevens et al., 2003). In addition, surface EMG is inappropriate for evaluating the activities of deep muscles such as the gluteus minimus.

Recently, the use of positron emission tomography (PET) and $\left[{ }^{18} \mathrm{~F}\right]$ fluorodeoxyglucose (FDG) has emerged as a more satisfactory method for investigating cumulative muscle activity during exercise and providing images of the spatial distribution of skeletal muscle metabolism (Fujimoto et al., 2000; Kemppainen et al., 2002; Oi et al., 2003; Shimada et al., 2007; Tashiro et al., 1999). FDG PET analysis is a metabolic imaging modality that involves the detection of intracellular FDG-6-P using gamma ray emission (Phelps et al., 1979). FDG is a glucose analog that is taken up by glucose using cells from the circulation through glucose transporters 1-4. FDG enters the glycolysis pathway and is phosphorylated into FDG-6-phosphate by hexokinase (Sokoloff et al., 1977). Intracellular FDG-6-P accumulates as it is a poor substrate for glucose-phosphate isomerase which converts glucose to fructose, and it therefore escapes dephosphorylation (Bessell \& Thomas, 1973). FDG can be used to assess cumulative muscle activity over an extended period of time because the half-life of $18 \mathrm{~F}$ is relatively long (109.8 $\mathrm{min}$ ) compared with that of other positron-emitting tracers; however, transient measurements are impossible. 
FDG PET is useful for comparing task-specific muscle activity because FDG uptake is closely correlated with exercise intensity (Fujimoto et al., 2003; Kemppainen et al., 2002; Pappas et al., 2001). Results of regression analyses between normalized biceps FDG uptake and the number of repetitions of elbow flexion performed with 2 and $10 \mathrm{lb}$ weights showed statistically significant positive correlations for both the $2 \mathrm{lb}$ and $10 \mathrm{lb}$ weights. The ratio of the slopes of the regression lines for the $10 \mathrm{lb}$ and $2 \mathrm{lb}$ weights was 4.94 , indicating an almost fivefold difference between the external forces produced by the elbow flexors for these two loads (Pappas et al., 2001).

In this chapter, we review the findings of previous studies to demonstrate the importance of FDG PET for exercise studies and the development of rehabilitation programs for the elderly.

\section{Glucose uptake in skeletal muscles during walking}

\subsection{Characteristics of gait function in older adults}

Healthy elderly people exhibit decreased muscle mass, strength, and power production compared with healthy young people (Gallagher et al., 1997; Klitgaard et al., 1990; Larsson et al., 1979; Lynch et al., 1999; Metter et al., 1997; Porter et al., 1997; Poulin et al., 1992; Thelen et al., 1996). These decreases result in a slower gait speed, shorter step length, shorter swing phase and less range of motion at the hip, knee, and ankle joints during walking (Crowinshield et al., 1978; Elble et al., 1991; Finley et al., 1969; Hageman \& Blanke, 1986; Judge et al., 1993, 1996; Kerrigan et al., 1998; Murray et al., 1969; Ostrosky et al., 1994; Winter et al., 1990). Reduced gait function in older people is associated with a decreased ability to undertake the activities of daily living (Brach \& VanSwearingen, 2002; Guralnik et al., 2000).

Kinesiological studies show that older adults perform locomotor tasks nearer their maximal torque-producing capabilities than young adults. This greater effort is associated with increased neural drive to the muscles responsible for walking and enhanced coactivation of opposing muscles (Hortobagyi \& DeVita, 2000; Hortobagyi et al., 2003). In addition, increased age is associated with a redistribution of joint torques and power as older adults use their hip extensors more and their knee extensors and ankle plantar flexors less when walking than the young. Data suggests that healthy older adults produce $279 \%$ more work at the hip, $39 \%$ less work at the knee, and $29 \%$ less work at the ankle compared with healthy young adults during gait (DeVita \& Hortobagyi, 2000). The localized increase in muscular activation in the elderly during sustained walking may cause decreased physical activity, not because of generalized exhaustion, but due to the onset of fatigue in particular muscles. Therefore, localized muscle energy expenditure is more important than global expenditure when considering control of movement in older people (O'Dwyer \& Neilson, 2000). The older adults also exposed gait instability due to enhanced coactivation of opposing muscles during walking.

\subsection{Differences in glucose metabolism between young and older adults during walking}

Shimada et al. (2009b) compared the differences between the glucose uptakes of skeletal muscles during walking in young and older adults using FDG PET. In this study, 10 healthy young and older men walked on a treadmill for $50 \mathrm{~min}$. Walking speed was maintained at $4.0 \mathrm{~km} / \mathrm{hr}$ for younger subjects and between 1.86 and $3.54 \mathrm{~km} / \mathrm{h}$ as achievable limits for 
older subjects. FDG (360 MBq) was injected 30 min after the start of walking. PET scans of the crista iliaca-planta region were conducted in six overlapping bed positions using a 7-min emission time per position and simultaneous attenuation correction. Glucose metabolism in the regions of interest (ROIs) was evaluated from the standardized uptake value (SUV) for FDG defined as follows:

$$
\mathrm{SUV}=\mathrm{C} / \mathrm{D} / \mathrm{w}
$$

where $\mathrm{C}(\mathrm{Bq} / \mathrm{ml})$ represents the concentration of radioactivity in the tissue, $\mathrm{D}(\mathrm{Bq})$ is the injected dose, and $\mathrm{w}$ is body mass (Sadato et al., 1998).

SUV was significantly increased in the semitendinosus, biceps femoris, iliacus, gluteus minimus, gluteus medius, and gluteus maximus muscles of older adults. FDG uptake ratios of older adults to young adults were 3.02 in the semitendinosus, 3.19 in the biceps femoris, 1.66 in the iliacus, 1.64 in the gluteus minimus, 3.68 in the gluteus medius, and 3.05 in the gluteus maximus muscles (Shimada et al., 2009b). The data indicate there was inefficient activity of these muscles during walking in the older adults. Figure 1 shows representative FDG PET images in a young and an older adult.
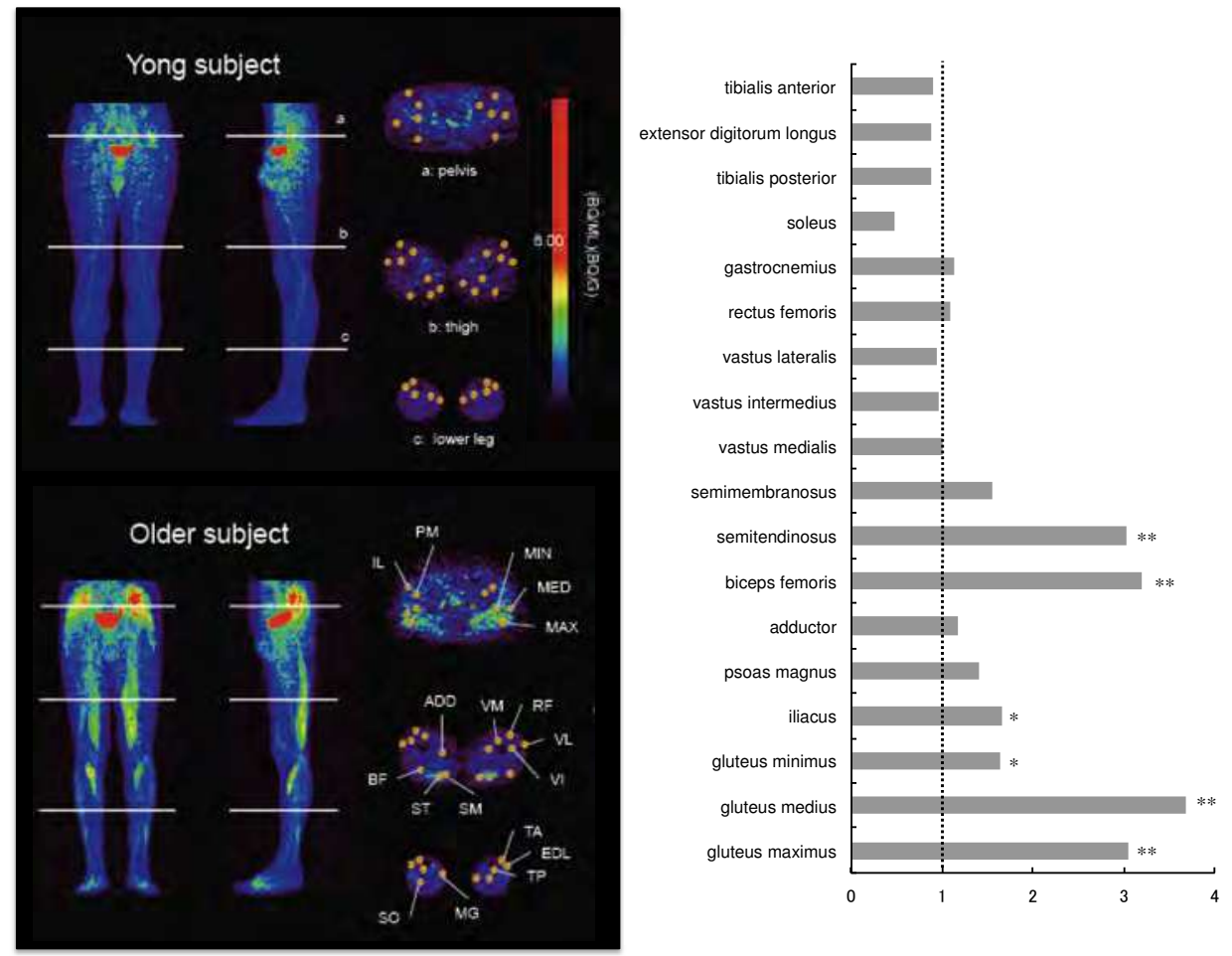

Fig. 1. FDG PET images taken after walking in a young and older subject.

Left panel: Projection and sectional images. The yellow circles indicate the following regions of interest: Section a (pelvis): $30 \mathrm{~mm}$ above the femoral head PM, psoas magnus; IL, iliacus; MIN, 
gluteus minimus; MED, gluteus medius; MAX, gluteus maximus. Section $b$ (tight): $50 \%$ of the distance from the femoral head to the knee joint RF, rectus femoris; VL, vastus lateralis; VI, vastus intermedius; VM, vastus medialis; $\mathrm{SM}$, semimembranosus; $\mathrm{ST}$, semitendinosus; $\mathrm{BF}$, biceps femoris; $\mathrm{AD}$, hip adductor. Section c (lower leg): $30 \%$ of the distance from the knee joint to the external malleolus TA, tibialis anterior; EDL, extensor digitorum longus; TP, tibialis posterior; SO, soleus; MG, medial gastrocnemius. The red color at the center of the pelvis resulted from the accumulation of FDG in the bladder. Right panel: Graph showing FDG uptake ratios of older adults to young adults. Significant difference: ${ }^{*}<0.05$ and ${ }^{* *} P<0.01$.

During walking, the hamstrings are most active from the period just before to just after heel contact. Before heel contact, the hamstrings decelerate knee extension to prepare for the placement of the foot on the ground. The hamstrings are active during the initial $10 \%$ of the stance phase in walking to assist with hip extension and to provide stability to the knee through coactivation. Strong activation of the gluteus maximus allows the hip to extend and prevents forward jackknifing of the torso at heel contact. The gluteus maximus remains active from heel contact to mid stance to support the weight of the body and produce hip extension. The iliacus becomes active before toe off to decelerate hip extension. Concentric muscle activation follows eccentric muscle activation to bring the hip into flexion just before toe off and during transition into initial swing. The gluteus medius and minimus, the primary hip abductors, are most active during single-limb support to stabilize the pelvis in the frontal plane (Neumann, 2002).

A previous study showed that hip, knee, and ankle joint muscles produce 44,5 , and $51 \%$ and 16,11 , and $73 \%$ of the total extensor work during the stance phase in older and younger adults, respectively (DeVita \& Hortobagyi, 2000). These data suggest that older adults perform similar amounts of work at the hip and ankle, but young adults perform the majority of work at the ankle (DeVita \& Hortobagyi, 2000). In addition, coactivation time of the thigh muscles is higher in older people than in young people, and there is a linear correlation between the coactivation time and the metabolic cost during walking (Mian et al., 2006). The redistribution of joint power during walking in older adults increases glucose metabolism in the hip extensors (gluteus maximus, hamstrings) and coactivation increases glucose metabolism of the hamstrings.

The hip joint is stabilized by the gluteus medius in the initial phase of the gait cycle and by the gluteus minimus during the mid- and late-phases (Gottschalk et al., 1989). However, the degree of activation of the gluteus minimus muscle during walking is unclear because activities of deep muscles such as the iliacus and gluteus minimus cannot be evaluated by EMG. By contrast, FDG PET can measure the activity of deep muscles. FDG PET analyses showed glucose metabolism in the gluteus minimus during walking in young adults was 2.1 times higher than that at rest and higher than that of the gluteus maximus, gluteus medius, and thigh muscles (Oi et al., 2003).

\section{Oxygen consumption and FDG uptake in skeletal muscles during exercise}

\subsection{Physical activity and oxygen consumption in older adults}

Many studies have shown that there is an association between restricted outdoor activities and the deterioration of physical function in healthy and frail older people (Bruce \& McNamara, 1992; Clarfield \& Bergman, 1991; Fujita et al., 2006; Ganguli et al., 1996; Kono \& 
Kanagawa, 2001; Kono et al., 2004). A two-year prospective study in initially able-bodied older individuals showed an association between a low frequency of baseline outdoor activity and incident disability (Fujita et al., 2006). In older individuals who went outdoors once a week or less, the adjusted risks of incident mobility impairment (odds ratio $=4.02$ ) and disability in instrumental activities of daily living (odds ratio $=2.65$ ) were significantly higher compared with an active group who went outside once a day or more. Outdoor activity may be restricted in individuals who have difficulty walking for extended periods (Simonsick et al., 2005).

Muscle activity during exercise results in a mechanical energy cost, which is reflected by whole body metabolic cost. During physical activity, there is relatively greater muscle activity and increased levels of coactivation of opposing muscles in older people (Hortobagyi \& DeVita, 2000; Hortobagyi et al., 2003). Furthermore, oxygen consumption $\left(\mathrm{VO}_{2}\right)$, which provides an index of walking efficiency, is greater in older adults even when there are no gait impairments (Malatesta et al., 2003; Martin et al., 1992; McCann \& Adams, 2002; Waters et al., 1988). These data suggest that older adults may have difficulties in performing the activities of daily living as they have to work at a higher level of effort relative to their maximum capability (Hortobagyi et al., 2003).

\subsection{Muscular activity and oxygen consumption}

Unlike other techniques, FDG PET allows the observation of continuous activities such as extended walking and can measure cumulative muscle metabolism during unrestricted physical activities. Furthermore, FDG uptake closely correlates with exercise intensity in healthy adults and can be used to compare task-specific muscle activity (Fujimoto et al., 2003; Kemppainen et al., 2002; Pappas et al., 2001).

\subsection{Relationship between FDG uptakes and $\mathrm{VO}_{2}$}

Few studies have investigated the relative contribution of different muscle groups to whole body energy consumption during walking. In one study, 10 community-dwelling older women participated in FDG PET and $\mathrm{VO}_{2}$ analyses during exercise on separate days within one week (Shimada et al., 2010). $\mathrm{VO}_{2}$ during walking was determined using an automated open-circuit gas analysis system (Cosmed $\mathrm{K}_{4} \mathrm{~b}^{2}$, Rome, Italy). The gas analyzers were calibrated immediately before each test using ambient air comprising certified standard gases at $15.94 \%$ oxygen and $4.97 \%$ carbon dioxide (Sumitomo Seika Chemicals Co., Ltd., Osaka, Japan). The subjects walked for $12 \mathrm{~min}$ at a comfortable speed on a circular $16 \mathrm{~m}$ indoor course and breath-by-breath data were obtained from the gas analyzers during walking, which was stored in the analyzer's memory. The mean $\mathrm{VO}_{2}$ values from the $3^{\text {rd }}$ to the $12^{\text {th }}$ min were used to assess constant whole body energy metabolism (McArdle et al., 1997). All SUV values were adjusted for the distance walked during the 50 min FDG PET trial as:

$$
\text { Adjusted SUV }=\mathrm{x} / \mathrm{a}
$$

where $x$ represents the measured SUV, and a represents the walking distance in $\mathrm{km}$.

The left and right panels show representative projection images of FDG PET uptake taken after walking. The scatterplot shows correlations between $\mathrm{VO}_{2}$ and adjusted SUV for different muscle groups. 
The $\mathrm{VO}_{2}$ during walking was significantly and positively correlated with the adjusted SUV in the biceps femoris, gluteus minimus, gluteus medius, and the pelvis muscle group (Fig. 2; Shimada et al., 2010). This shows that these muscle groups contribute to the increase in $\mathrm{VO}_{2}$ during walking in older adults. Evidence suggests the hamstrings, including biceps femoris, are most active from a period just before to just after heel contact during walking (Neumann, 2002). Furthermore, older adults display higher levels of antagonist coactivation during gross locomotor tasks (Hortobagyi \& DeVita, 2000; Mian et al., 2006). Indeed, there is greater antagonist thigh muscle coactivation during walking in older men than young men and a linear relationship between muscle coactivation and whole body metabolic cost of walking (Mian et al., 2006).
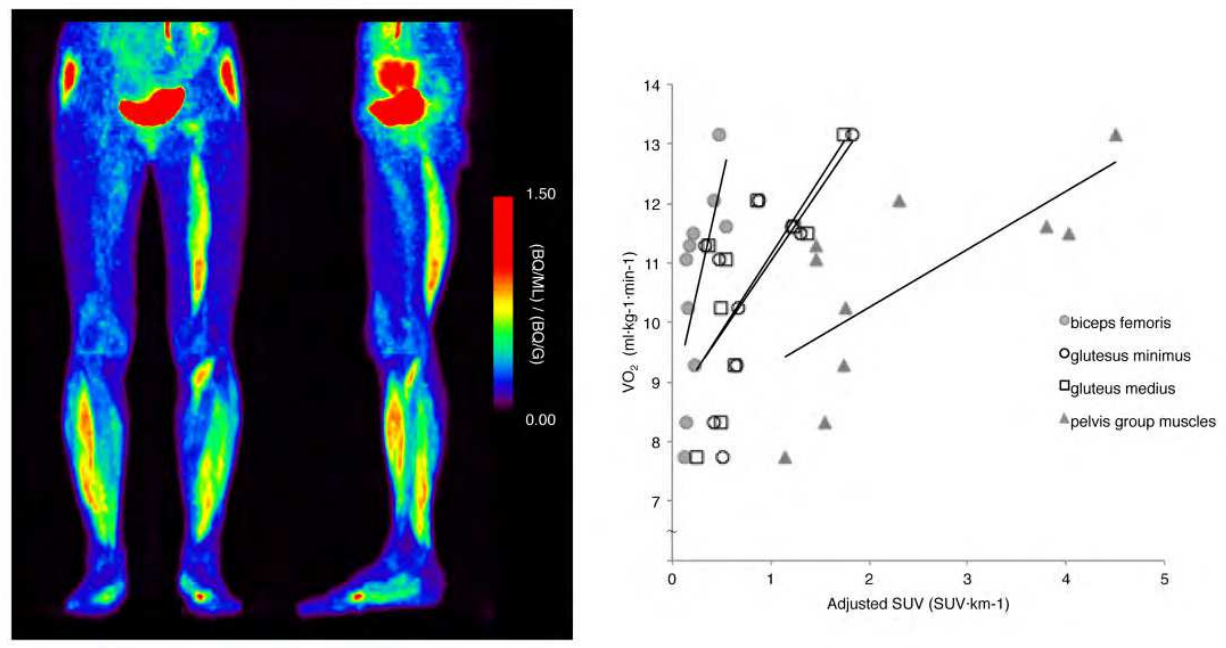

Fig. 2. Relationship between glucose uptake and $\mathrm{VO}_{2}$ in older subjects.

Gait endurance contributes to the ability to perform the activities of daily living; however, information describing the fatiguing effect of daily activities on gait is limited. Previous studies indicate there is a relationship between muscle fatigue and physical function (Gribble \& Hertel, 2004a,b; Helbostad et al.,2007; Kavanagh et al., 2006) as there are changes in gait step width, mediolateral trunk acceleration amplitude, step-length variability, and trunk acceleration variability in the vertical direction following physical fatigue induced by an atypical daily activity (i.e., sit-to-stand task), even in the absence of a change in gait speed (Helbostad et al.,2007). These gait variables are related to the incidence of falling in older people (Hausdroff et al., 2001; Maki, 1997; Mbourou et al, 2003).

The primary hip abductors, the gluteus medius and minimus, stabilize the pelvis in the frontal plane and are important for single-limb support (Neumann, 2002). During walking, the body is unstable for most of the stride cycle as its center of mass is outside the base of support $80 \%$ of the time (Winter, 1991). During walking in older people, mediolateral stability is generally reduced resulting in greater activation of the hip abductors compared with the young (Dean et al., 2007). Reduced mediolateral stability in older people can lead to an increased step width to avoid falling while walking at a preferred speed; a wider step 
width increases both mechanical work and metabolic energy expenditure (Donelan et al., 2001). A previous study showed older subjects selected a narrower step width when walking at a preferred speed on a treadmill with external lateral stabilization, and the energy cost with lateral stabilization was reduced in a zero step width condition i.e., tandem-like walking, compared with without stabilization (Dean et al., 2007). These findings are supported by observations of a significant relationship between the adjusted SUV in the hip abductors and $\mathrm{VO}_{2}$.

A previous study showed that FDG uptakes by the biceps femoris, semitendinosus, iliacus, gluteus minimus, gluteus medius, and gluteus maximus muscles were significantly increased in older adults compared with young subjects (Shimada et al. 2009b). However, lower leg muscles such as tibialis anterior, tibialis posterior, and soleus had a ratio of less than one. These results suggest that the excess muscle activity of the larger thigh muscles contributes to the increase in $\mathrm{VO}_{2}$ during walking in older adults.

Moreover, subjects with proportionally greater activity in their hip muscles had a higher $\mathrm{VO}_{2}$ while walking compared with those with proportionally lower hip muscle activity. There may be a redistribution of muscle activity with aging; studies show increased work by the hip musculature in older adults is associated with decreased work by the musculature of the more distal joints (e.g. reduced power in plantar flexor) in both healthy older adults (DeVita \& Hortobagyi, 2000; Judge et al., 1996) and older adults with a lower extremity disability (McGibbon \& Krebs, 2002; McGibbon et al., 2001). FDG PET findings provide further evidence describing increased output by the hip musculature and introduce new information indicating that this increased output increases $\mathrm{VO}_{2}$ in older adults during walking.

\section{Evaluation of a walking aid using FDG PET}

\subsection{Importance of walking aids in older adults}

Physical function is associated with the ability to perform the activities necessary for independent living without substantial risk of injury (Guralnik \& Simonsick, 1993). Evidence suggests that impaired gaits in the elderly strongly affect their ability to perform daily activities (Brach \& VanSwearingen, 2002; Guralnik et al., 2000); therefore, evaluation of gaits is an essential part of geriatric health and intervention programs. Kinesiological and epidemiological studies have shown that changes in gait scores such as decreased walking speed and stride length are associated with advanced age (Elble et al.,1991; Judge et al., 1996; Murray et al., 1969; Nagasaki et al., 1996; Nigg et al., 1994; Ostrosky et al., 1994; Winter et al., 1990). The age-related change in gait is characterized by a reduction in ankle power output during the terminal stance phase probably due to an age-related impairment in the power-generating capacity of the ankle which limits walking speed and stride length (McGibbon, 2003). In addition, the metabolic cost of walking is greater for the elderly than for young adults, even when there are no gait impairments (Malatesta et al., 2003; Martin et al., 1992; McCann \& Adams, 2002; Waters et al., 1988). The increased metabolic cost of walking can impair the activity and quality of life of elderly people as a decrease in physical activity rapidly degrades physical and psychological functions (Backmand et al., 2006; Young et al., 1995). 
There are many interventions that improve gait performance in non-disabled and disabled elderly people. Almost all these interventions include exercise programs to improve muscle strength or balance (Gillespie et al., 2003; Latham et al., 2003). Endurance in elderly people can also be improved by assisted devices such as canes or braces which affect gait speeds, stride lengths, and stability (Alexander, 1996; Joyce \& Kirby, 1991; Kuan et al., 1999; Roomi et al., 1998; Van Hook et al., 2003). Shimada et al. developed an automated stride assistance system (Shimada et al., 2009a) (SAS) (Honda R \& D Co. Ltd., Wako, Japan) (Figure 3), which uses robotic engineering to control walk ratios (stride length/cadence) and add supporting power to the thigh during walking. The SAS weighs $3.5 \mathrm{~kg}$ and it was developed to teach walking efficiency and improve gait endurance in elderly people with age-related short stride length. However, the SAS is limited because it supports movement of the hip joint during walking, which can lead to deterioration of muscle activity in the lower extremities during long-term intervention studies or practical applications.

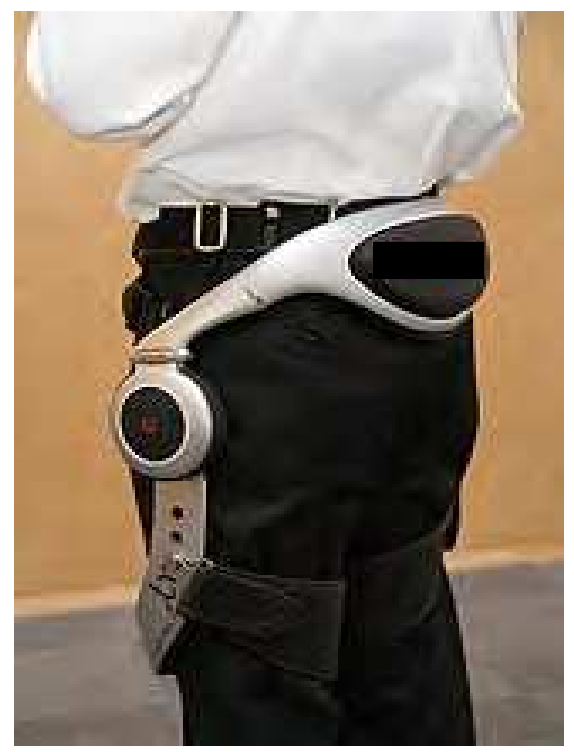

Fig. 3. Stride assistance system that can control walk ratios (stride length/cadence) and support the thigh during walking.

\subsection{Muscular metabolism during walking using a robotic stride assistance system}

FDG PET has been used to evaluate muscular activity during exercise with a stride assistance system (Shimada et al., 2007, 2008). In this research, 10 healthy younger men (mean age, 24.1 years) and 7 healthy older men (mean age, 76.0 years) completed FDG PET measurements twice after walking with and without the SAS. The sequence of the experiments was randomized to negate the confounding effect of prior experience of walking on a treadmill. Subjects were asked to walk for $50 \mathrm{~min}$ at $4.0 \mathrm{~km} / \mathrm{h}$ on a treadmill (MAT-5500; Fukuda Denshi Co. Ltd., Tokyo, Japan). All young subjects walked at the target speed of $4.0 \mathrm{~km} / \mathrm{h}$. The speed of the treadmill was adjusted to $2.89-3.82 \mathrm{~km} / \mathrm{h}$ without the SAS and $3.03-4.03 \mathrm{~km} / \mathrm{h}$ with the SAS for the older subjects who could walk at a constant speed. 
Figure 4 shows representative FDG PET images taken in young and older subjects after walking with or without the SAS. Glucose utilization in the lower-extremity muscles was evident after walking. In young subjects, walking with the SAS significantly increased FDG uptakes by the tibialis posterior and the medial gastrocnemius compared with walking without it (Shimada et al., 2007). FDG uptake ratios (SUV after walking with the SAS: SUV after walking without the SAS) of the tibialis posterior and medial gastrocnemius were 2.13 and 2.36, respectively. Walking with the SAS did not have significant effects on any other muscles. In older adults, there were no significant differences between the SUVs with and without the SAS in all lower-extremity muscles. However, walking speeds (mean walking speed without SAS, $3.46 \mathrm{~km} / \mathrm{h}$; mean walking speed with SAS, $3.56 \mathrm{~km} / \mathrm{h}$ ) and stride lengths (mean stride length without SAS, $54.9 \mathrm{~cm}$, mean stride length with SAS, $58.2 \mathrm{~cm}$ ) were

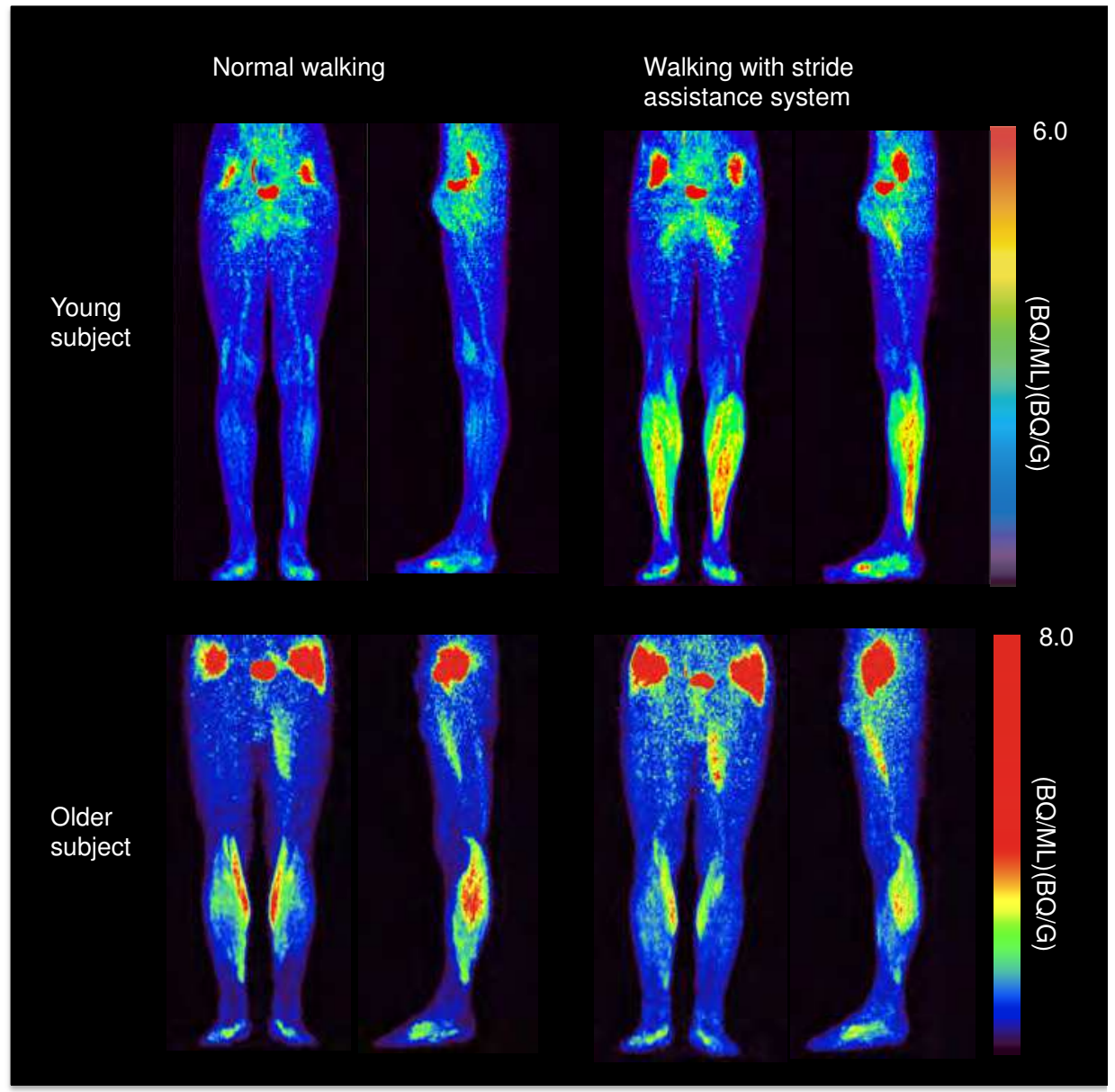

Fig. 4. FDG PET images taken after walking without or with the SAS.

The left and right panels show projection images taken after walking without or with the SAS, respectively. 
significantly increased when walking on the treadmill with the SAS in the older subjects. These results suggest that the SAS can facilitate efficient walking patterns irrespective of muscle activity. The SAS may have provided assistance to the thigh and increased the torque of the hip of the older subjects resulting in improved walking scores. Therefore, stride length and walking speed increased without activating lower-extremity muscle activity.

\section{Evaluation of exercise intervention using FDG PET}

\subsection{Effects of exercise in older adults}

Older people enjoy walking exercise because it is familiar and more convenient than many other sports and recreational activities (Morris \& Hardman, 1997; Mutrie \& Hannah, 2004). Intervention studies show that strength or endurance training in older people can improve measures of gait such as walking speed (Binder et al., 2004; Brown \& Holloszy, 1993; Buchner et al., 1997a; Ettinger et al., 1997; Judge et al., 1993). Endurance training also improves physical fitness, particularly cardiovascular fitness, as well as cognitive functions (van Uffelen et al., 2008). The development of targeted exercise programs may be facilitated by exercise intervention studies that allow a better understanding of the effects of muscle activity during walking. However, results from previous intervention studies investigating the effects of strength or endurance training on walking speed and gait are inconclusive (Buchner et al., 1997b; Ettinger et al., 1997). Walking is a near-perfect exercise for healthy and frail older people (Morris \& Hardman, 1997); therefore, if walking induces walkingspecific adaptations, interventions involving walking may be an efficient and appropriate means of improving walking function in older people (Shimada et al., 2003). However, knowledge of the effects of walking exercise on the physical performance of older people is limited. Most walking exercise interventions are prescribed in combination with exercises aimed at increasing muscle strength, neuromuscular coordination, and balance (Morris et al., 1999), and physical performance is not always assessed in intervention studies (Ebrahim et al., 1997).

\subsection{Comparison of FDG uptakes in skeletal muscles before and after intervention}

The functionality of walking may be improved by increasing the stride length of older people, which in turn may result in benefits such as improved walking efficiency. These benefits may supersede those derived from improved aerobic fitness alone. Shimada et al. (2009a) assessed the effects of a walking program for the elderly using the SAS. Fifteen subjects participated in a three-month walking program of two 90-min supervised sessions per week using the SAS. For FDG PET analysis, subjects walked for $50 \mathrm{~min}$ at a comfortable speed on a circular indoor walking track without the SAS. Figure 5 shows representative FDG PET images taken before and after the exercise intervention (Shimada et al., 2009a). FDG uptakes by the gluteus minimus, gluteus medius, and rectus femoris were significantly lower after the intervention than before, although walking speed during FDG PET measurements increased after the intervention. In contrast, the medial gastrocnemius and soleus (the lower distal muscles) showed higher FDG uptakes after the intervention than before, although the difference was not statistically significant. 


\section{Before intervention}

\section{After intervention}

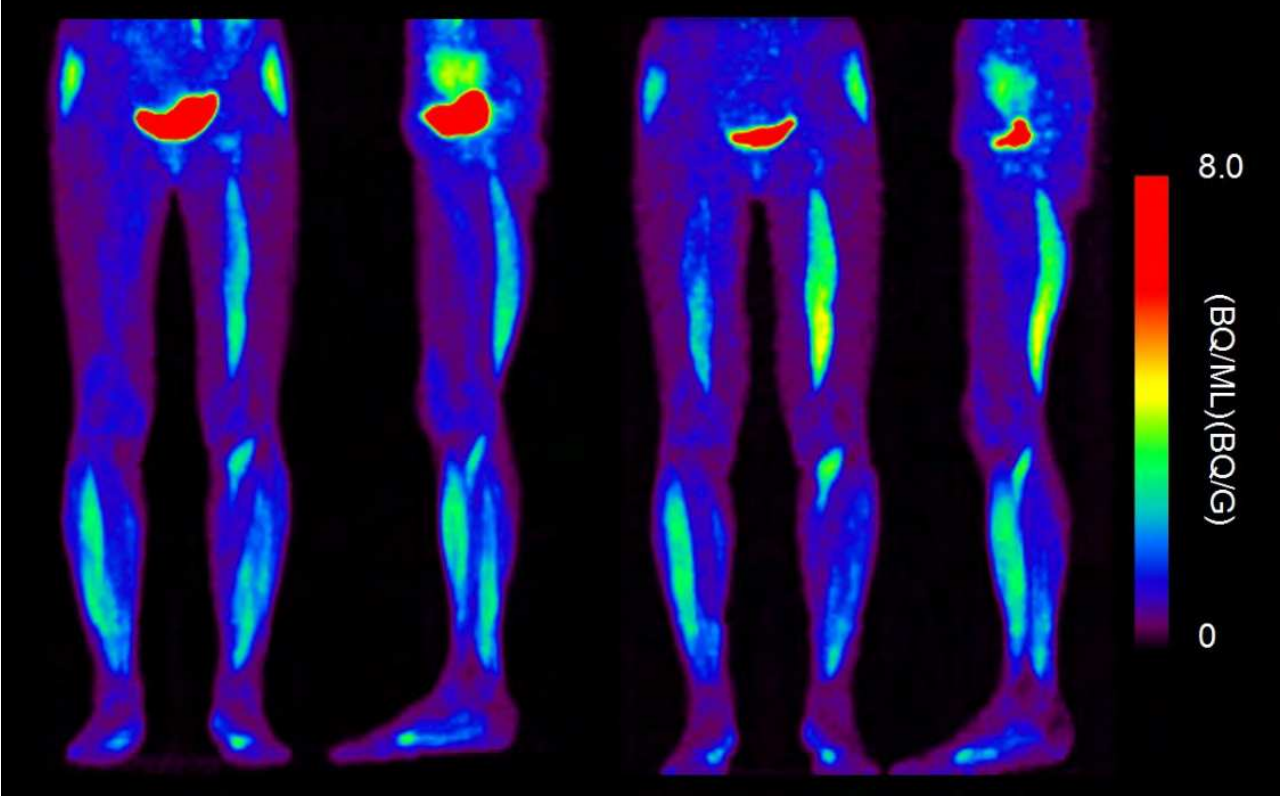

Fig. 5. FDG PET images of an older woman before and after the intervention.

The left and right panels show before and after intervention images, respectively. FDG uptakes by the gluteus minimus, gluteus medius, rectus femoris and pelvic muscles after the intervention were significantly lower than before.

The gluteus medius and gluteus minimus are the two primary hip abductors. They are most active during the first $40 \%$ of the gait cycle and they stabilize the pelvis in the frontal plane (Neumann, 2002). It is possible that the activity of the hip abductors decreases to improve mediolateral stability during long-distance walking. Further studies must be carried out using kinematic and kinetic analyses to fully understand the mechanisms involved in the change in cumulative muscle activity during prolonged walking.

Previous data indicate that the activity of hip-related muscles and hamstrings is greater than that of other lower extremity muscles in elderly people during walking. Shimada et al. 2009a found a significant decrease in the activity of the pelvic muscles (iliacus and gluteus muscles) during intervention with the SAS. However, the intervention did not increase FDG uptake of the soleus and gastrocnemius muscles, suggesting that the walking intervention improved the efficiency of muscle activity but did not redistribute muscular effort. This indicates that a walking intervention with the SAS has potential to increase walking endurance in the elderly. Indeed, the distance walked in $50 \mathrm{~min}$ after the intervention (median, $3579.0 \mathrm{~m}$ ) was greater than that before the intervention (median, $3051.0 \mathrm{~m}$ ); however, this difference was not statistically significant. 
The quadriceps femoris controls knee flexion and acts as a shock absorber after heel contact; it then supports the weight of the body in mid-stance. The rectus femoris differs from that of other knee extensors as it is a hip flexor and its activity increases immediately after the toeoff phase (Neumann, 2002). A previous study showed that antagonist thigh muscle coactivation (e.g., activation of the vastus medialis, vastus lateralis, and biceps femoris) is $31 \%$ greater in older than in younger adults, and coactivation is moderately correlated with the metabolic cost of walking (Mian et al., 2006). The SAS automatically lends horizontal force to the thigh to facilitate an optimal walk ratio and may teach elderly people to use their muscles more efficiently. The consecutive stimuli provided by the SAS may help elderly people adopt an efficient walking pattern.

\section{Functional FDG PET imaging as evaluating of frailty}

Frail elderly people are particularly vulnerable for developing disabilities (Boyd et al., 2005; Gill et al., 2004; Hardy et al., 2005) and are at an increased risk for falls, disabilities, hospitalization, institutionalization and death, compared with their age-matched non-frail counterparts (Espinoza \& Walston, 2005). Disability is closely related to medical spending; therefore, prevention of disability can lead to reduced health care costs (Cutler, 2001). Physical frailty indicators include mobility, strength, endurance, nutrition, physical inactivity, balance, and motor processing (Ferrucci et al., 2004). Gait disorder is a particularly important indicator of frailty and an independent predictor of disability. The findings of research using FDG PET has revealed a cycle of gait disorder (Figure 6).

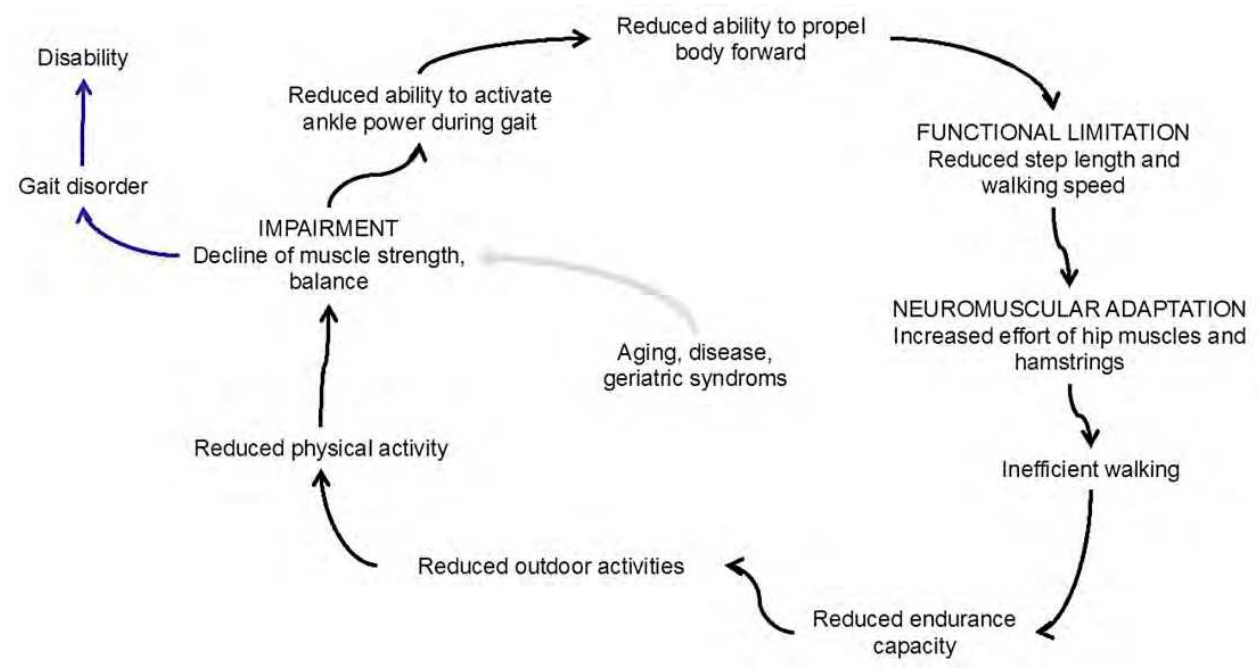

Fig. 6. Schematic diagram of the incidence of gait disorder in older adults.

Aging results in impaired muscle strength and balance, which reduces the ability to activate the ankle plantar muscles during gait and propel the body forward. These impairments manifest as functional limitations including reduced step length and walking speed, which are compensated for by neuromuscular adaptations such as increased effort of the hip 
muscles and hamstrings. Walking becomes inefficient and there is reduced endurance capacity. This leads to decreased involvement in outdoor activities and therefore physical activity, which worsens the impaired muscle strength and balance. Ultimately, the cycle can lead to gait disorder and disability.

\section{Conclusions}

FDG PET has proved useful for understanding the ability of older adults to perform physical activities. Because FDG uptake is closely correlated with exercise intensity, it can be used for comparing task-specific muscle activity. FDG PET and $\mathrm{VO}_{2}$ analyses indicate that older adults may have difficulties in performing the activities of daily living as they have to work at a higher level of effort relative to their maximum capability due to a redistribution of muscle activity with aging. Automated exercise intervention, such as the automated stride assistance system (SAS), may help slow the cycle of events that ultimately can lead to gait disorder and disability. FDG PET evaluation of glucose metabolism in the muscles of the elderly following intervention with the automated SAS indicates that the SAS has the potential to increase walking endurance. We suggest that FDG PET is a useful method to evaluate the effects of interventions and therefore develop rehabilitation programs.

\section{Acknowledgments}

This work received financial support from a Grant-in-Aid for Scientific Research (B) (Tokyo, Japan). The funding source had no role in the study design, data collection, analysis, or writing of this manuscript.

\section{References}

Alexander, N.B. Gait disorders in older adults. J Am Geriatr Soc. 1996;44: 434-451.

Axel, L., \& Dougherty, L. MR imaging of motion with spatial modulation of magnetization. Radiology. 1989;171: 841-845.

Backmand, H., Kaprio, J., Kujala, U.M., Sarna, S., \& Fogelholm, M. Physical and psychological functioning of daily living in relation to physical activity. A longitudinal study among former elite male athletes and controls. Aging Clin Exp Res. 2006;18: 40-49.

Bessell, E.M., \& Thomas P. The effect of substitution at C-2 of D-glucose 6-phosphate on the rate of dehydrogenation by glucose 6-phosphate dehydrogenase (from yeast and from rat liver). Biochem J. 1973;131: 83-89.

Binder, E.F., Brown, M., Sinacore, D.R., Steger-May, K., Yarasheski, K.E., \& Schechtman, K.B. Effects of extended outpatient rehabilitation after hip fracture: A randomized controlled trial. JAMA. 2004;292: 837-846.

Boyd, C.M., Xue, Q.L., Simpson, C.F., Guralnik, J.M., \& Fried, L.P. Frailty, hospitalization, and progression of disability in a cohort of disabled older women. The American Journal of Medicine. 2005;118: 1225-1231.

Brach, J.S., \& VanSwearingen, J.M. Physical impairment and disability: relationship to performance of activities of daily living in community-dwelling older men. Phys Ther. 2002;82: 752-761. 
Brown, M., \& Holloszy, J.O. Effects of walking, jogging and cycling on strength, flexibility, speed and balance in 60- to 72-year olds. Aging (Milano). 1993;5: 427-434.

Bruce, M.L., \& McNamara, R. Psychiatric status among the homebound elderly: an epidemiologic perspective. J Am Geriatr Soc. 1992;40: 561-566.

Buchner, D.M., Cress, M.E., de Lateur, B.J., et al. A comparison of the effects of three types of endurance training on balance and other fall risk factors in older adults. Aging (Milano). 1997a;9: 112-119.

Buchner, D.M., Cress, M.E., de Lateur, B.J., et al. The effect of strength and endurance training on gait, balance, fall risk, and health services use in community-living older adults. J Gerontol A Biol Sci Med Sci. 1997b;52: M218-224.

Clarfield, A.M., \& Bergman, H. Medical home care services for the housebound elderly. CMAJ. 1991;144: 40-45.

Crowinshield, R.D., Brand, R.A., \& Johnston, R.C. The effects of walking velocity and age on hip kinematics and kinetics. Clin Orthop Relat Res. 1978: 140-144.

Cutler, D.M. Declining disability among the elderly. Health Aff (Millwood). 2001;20: 11-27.

Dean, J.C., Alexander, N.B., \& Kuo, A.D. The effect of lateral stabilization on walking in young and old adults. IEEE Trans Biomed Eng. 2007;54: 1919-1926.

DeVita, P., \& Hortobagyi, T. Age causes a redistribution of joint torques and powers during gait. J Appl Physiol. 2000;88: 1804-1811.

Donelan, J.M., Kram, R., \& Kuo, A.D. Mechanical and metabolic determinants of the preferred step width in human walking. Proc R Soc Lond B. 2001;268: 1985-1992.

Drace, J.E., \& Pelc, N.J. Measurement of skeletal muscle motion in vivo with phase-contrast MR imaging. J Magn Reson Imaging. 1994;4: 157-163.

Ebrahim, S., Thompson, P.W., Baskaran, V., \& Evans, K. Randomized placebo-controlled trial of brisk walking in the prevention of postmenopausal osteoporosis. Age Ageing. 1997;26: 253-260.

Elble, R.J., Thomas, S.S., Higgins, C., \& Colliver, J. Stride-dependent changes in gait of older people. J Neurol. 1991;238: 1-5.

Espinoza, S., \& Walston, J.D. Frailty in older adults: insights and interventions. Cleveland Clinic Journal of Medicine. 2005;72: 1105-1112.

Ettinger, W.H., Jr., Burns, R., Messier, SP., et al. A randomized trial comparing aerobic exercise and resistance exercise with a health education program in older adults with knee osteoarthritis. The Fitness Arthritis and Seniors Trial (FAST). JAMA. 1997;277: 25-31.

Ferrucci, L., Guralnik, J.M., Studenski, S., Fried, L.P., Cutler, G.B., Jr., \& Walston, J.D. Designing randomized, controlled trials aimed at preventing or delaying functional decline and disability in frail, older persons: a consensus report. Journal of the American Geriatrics Society. 2004;52: 625

Finley, F.R., Cody, K.A., \& Finizie, R.V. Locomotion patterns in elderly women. Arch Phys Med Rehabil. 1969;50: 140-146.

Fujimoto, T., Itoh, M., Tashiro, M., Yamaguchi, K., Kubota, K., \& Ohmori, H. Glucose uptake by individual skeletal muscles during running using whole-body positron emission tomography. Eur J Appl Physiol. 2000;83: 297-302.

Fujimoto, T., Kemppainen, J., Kalliokoski, K.K., Nuutila, P., Ito, M., \& Knuuti, J. Skeletal muscle glucose uptake response to exercise in trained and untrained men. Med Sci Sports Exerc. 2003;35: 777-783. 
Fujita, K., Fujiwara, Y., Chaves, P.H., Motohashi, Y., \& Shinkai, S. Frequency of going outdoors as a good predictor for incident disability of physical function as well as disability recovery in community-dwelling older adults in rural Japan. J Epidemiol. 2006;16: 261-270.

Gallagher, D., Visser, M., De Meersman, R.E., et al. Appendicular skeletal muscle mass: effects of age, gender, and ethnicity. J Appl Physiol. 1997;83: 229-239.

Ganguli, M., Fox, A., Gilby, J., \& Belle, S. Characteristics of rural homebound older adults: A community-based study. J Am Geriatr Soc. 1996;44: 363-370.

Gill, T.M., Allore, H., Holford, T.R., \& Guo, Z. The development of insidious disability in activities of daily living among community-living older persons. The American Journal of Medicine. 2004;117: 484-491.

Gillespie, L.D., Gillespie, W.J., Robertson, M.C., Lamb, S.E., Cumming, R.G., \& Rowe, B.H. Interventions for preventing falls in elderly people. Cochrane Database Syst Rev. 2003: CD000340.

Gottschalk, F., Kourosh, S., \& Leveau, B. The functional anatomy of tensor fasciae latae and gluteus medius and minimus. J Anat. 1989;166: 179-189.

Gribble, P.A., \& Hertel, J. Effect of lower-extremity muscle fatigue on postural control. Arch Phys Med Rehabil. 2004a;85: 589-592.

Gribble, P.A., \& Hertel, J. Effect of hip and ankle muscle fatigue on unipedal postural control. J Electromyogr Kinesiol. 2004b;14: 641-646.

Guralnik, J.M., Ferrucci, L., Pieper, C.F., et al. Lower extremity function and subsequent disability: consistency across studies, predictive models, and value of gait speed alone compared with the short physical performance battery. J Gerontol A Biol Sci Med Sci. 2000;55: M221-231.

Guralnik, J.M., \& Simonsick, E.M. Physical disability in older Americans. J Gerontol. 1993;48 Spec No: 3-10.

Hageman, P.A., \& Blanke, D.J. Comparison of gait of young women and elderly women. Phys Ther. 1986;66: 1382-1387.

Hardy, S.E., Dubin, J.A., Holford, T.R., \& Gill, T.M. Transitions between states of disability and independence among older persons. American Journal of Epidemiology. 2005;161: 575-584.

Hausdorff, J.M., Rios, D.A., \& Edelberg, H.K. Gait variability and fall risk in communityliving older adults: A 1-year prospective study. Arch Phys Med Rehabil. 2001;82: 1050-1056.

Helbostad, J.L., Leirfall, S., Moe-Nilssen, R., \& Sletvold, O. Physical fatigue affects gait characteristics in older persons. J Gerontol A Biol Sci Med Sci. 2007;62: 1010-1015.

Hortobagyi, T., \& DeVita, P. Muscle pre- and coactivity during downward stepping are associated with leg stiffness in aging. J Electromyogr Kinesiol. 2000;10: 117-126.

Hortobagyi, T., Mizelle, C., Beam, S., \& DeVita, P. Old adults perform activities of daily living near their maximal capabilities. J Gerontol A Biol Sci Med Sci. 2003;58: M453460.

Joyce, B.M., \& Kirby, R.L. Canes, crutches and walkers. Am Fam Physician. 1991;43: 535-542.

Judge, J.O., Davis, R.B., 3rd, \& Ounpuu, S. Step length reductions in advanced age: the role of ankle and hip kinetics. J Gerontol A Biol Sci Med Sci. 1996;51: M303-312.

Judge, J.O., Underwood, M., \& Gennosa, T. Exercise to improve gait velocity in older persons. Arch Phys Med Rehabil. 1993;74: 400-406. 
Kavanagh, J.J., Morrison, S., \& Barrett, R.S. Lumbar and cervical erector spinae fatigue elicit compensatory postural responses to assist in maintaining head stability during walking. J Appl Physiol. 2006;101: 1118-1126.

Kemppainen, J., Fujimoto, T., Kalliokoski, K.K., Viljanen, T., Nuutila, P., \& Knuuti, J. Myocardial and skeletal muscle glucose uptake during exercise in humans. $J$ Physiol. 2002;542: 403-412.

Kerrigan, D.C., Todd, M.K., Della Croce, U., Lipsitz, L.A., \& Collins, J.J. Biomechanical gait alterations independent of speed in the healthy elderly: Evidence for specific limiting impairments. Arch Phys Med Rehabil. 1998;79: 317-322.

Klitgaard, H., Mantoni, M., Schiaffino, S., et al. Function, morphology and protein expression of ageing skeletal muscle: A cross-sectional study of elderly men with different training backgrounds. Acta Physiol Scand. 1990;140: 41-54.

Kono, A., Kai, I., Sakato, C., \& Rubenstein, L.Z. Frequency of going outdoors: a predictor of functional and psychosocial change among ambulatory frail elders living at home. $J$ Gerontol A Biol Sci Med Sci. 2004;59: 275-280.

Kono, A., \& Kanagawa, K. Characteristics of housebound elderly by mobility level in Japan. Nurs Health Sci. 2001;3: 105-111.

Kuan, T.S., Tsou, J.Y., \& Su, F.C. Hemiplegic gait of stroke patients: the effect of using a cane. Arch Phys Med Rehabil. 1999;80: 777-784.

Larsson, L., Grimby, G., \& Karlsson, J. Muscle strength and speed of movement in relation to age and muscle morphology. J Appl Physiol. 1979;46: 451-456.

Latham, N., Anderson, C., Bennett, D., \& Stretton, C. Progressive resistance strength training for physical disability in older people. Cochrane Database Syst Rev. 2003: CD002759

Lynch, N.A., Metter, E.J., Lindle, R.S., et al. Muscle quality. I. Age-associated differences between arm and leg muscle groups. J Appl Physiol. 1999;86: 188-194.

Malatesta, D., Simar, D., Dauvilliers, Y., et al. Energy cost of walking and gait instability in healthy 65- and 80-yr-olds. J Appl Physiol. 2003;95: 2248-2256.

Maki, B.E. Gait changes in older adults: Predictors of falls or indicators of fear. J Am Geriatr Soc. 1997;45: 313-320.

Martin, P.E., Rothstein, D.E., \& Larish, D.D. Effects of age and physical activity status on the speed-aerobic demand relationship of walking. J Appl Physiol. 1992;73: 200-206.

Mbourou, G.A., Lajoie, Y., \& Teasdale, N. Step length variability at gait initiation in elderly fallers and non-fallers, and young adults. Gerontology. 2003;49: 21-26.

McArdle, D.W., Katch, I.F., \& Katch, L.V. (1997). Exercise Physiology: Energy, Nutrition, and Human Performance. 4th ed. Williams \& Wilkins, Baltimore, Maryland.

McCann, D.J., \& Adams, W.C. A dimensional paradigm for identifying the size-independent cost of walking. Med Sci Sports Exerc. 2002;34: 1009-1017.

McGibbon, C.A. Toward a better understanding of gait changes with age and disablement: Neuromuscular adaptation. Exerc Sport Sci Rev. 2003;31: 102-108.

McGibbon, C.A., \& Krebs, D.E. Compensatory gait mechanics in patients with unilateral knee arthritis. J Rheumatol. 2002;29: 2410-2419.

McGibbon, C.A., Krebs, D.E., \& Puniello, M.S. Mechanical energy analysis identifies compensatory strategies in disabled elders' gait. J Biomech. 2001;34: 481-490.

Metter, E.J., Conwit, R., Tobin, J., \& Fozard, J.L. Age-associated loss of power and strength in the upper extremities in women and men. J Gerontol A Biol Sci Med Sci. 1997;52: B267-276. 
Mian, O.S., Thom, J.M., Ardigo, L.P., Narici, M.V., \& Minetti, A.E. Metabolic cost, mechanical work, and efficiency during walking in young and older men. Acta Physiol (Oxf). 2006;186: 127-139.

Morris, J.N., Fiatarone, M., Kiely, D.K., et al. Nursing rehabilitation and exercise strategies in the nursing home. J Gerontol A Biol Sci Med Sci. 1999;54: M494-500.

Morris, J.N., \& Hardman, A.E. Walking to health. Sports Med. 1997;23: 306-332.

Murray, M.P., Kory, R.C., \& Clarkson, B.H. Walking patterns in healthy old men. J Gerontol. 1969;24: 169-178.

Mutrie, N., \& Hannah, M-K. Some work hard while others play hard. The achievement of current recommendations for physical activity levels at work, at home, and in leisure time in the west of Scotland. Int J Health Promot Educ. 2004;42: 109-107.

Nagasaki, H., Itoh, H., Hashizume, K., Furuna, T., Maruyama, H., \& Kinugasa, T. Walking patterns and finger rhythm of older adults. Percept Mot Skills. 1996;82: 435-447.

Neumann, D.A. (2002). Kinesiology of the musculoskeletal system: foundations for physical rehabilitation. St. Louis, Missouri, Mosby.

Nigg, B.M., Fisher, V., \& Ronskey, J.L. Gait characteristics as a function of age and gender. Gait Post 1994;2: 213-220.

O'Dwyer, N.J., \& Neilson, P.D. (2000). Metabolic energy expenditure and accuracy in movement: Relation to levels of muscle and cardiorespiratory activation and the sense of effort. In: Energetics of human activity. Human Kinetics. W.A. Sparrow, (Ed.), 1-42, Champaign, IL.

Oi, N., Iwaya, T., Itoh, M., Yamaguchi, K., Tobimatsu, Y., \& Fujimoto, T. FDG-PET imaging of lower extremity muscular activity during level walking. J Orthop Sci. 2003;8: 5561.

Ostrosky, K.M., VanSwearingen, J.M., Burdett, R.G., \& Gee, Z. A comparison of gait characteristics in young and old subjects. Phys Ther. 1994;74: 637-644; discussion 644-636.

Pappas, G.P., Olcott, E.W., \& Drace, J.E. Imaging of skeletal muscle function using 18FDG PET: Force production, activation, and metabolism. J Appl Physiol. 2001;90: 329-337.

Phelps, M.E., Huang, S.C., Hoffman, E.J., Selin, C., Sokoloff, L., \& Kuhl, D.E. Tomographic measurement of local cerebral glucose metabolic rate in humans with (F-18)2fluoro-2-deoxy-D-glucose: Validation of method. Ann Neurol. 1979;6: 371-388.

Pipe, J.G., Boes, J.L., \& Chenevert, T.L. Method for measuring three-dimensional motion with tagged MR imaging. Radiology. 1991;181: 591-595.

Porter, M.M., Vandervoort, A.A., \& Kramer, J.F. Eccentric peak torque of the plantar and dorsiflexors is maintained in older women. J Gerontol A Biol Sci Med Sci. 1997;52: B125-131.

Poulin, M.J., Vandervoort, A.A., Paterson, D.H., Kramer, J.F., \& Cunningham, D.A. Eccentric and concentric torques of knee and elbow extension in young and older men. Can J Sport Sci. 1992;17: 3-7.

Roomi, J., Yohannes, A.M., \& Connolly, M.J. The effect of walking aids on exercise capacity and oxygenation in elderly patients with chronic obstructive pulmonary disease. Age Ageing. 1998;27: 703-706.

Sadato, N., Tsuchida, T., Nakaumra, S., et al. Non-invasive estimation of the net influx constant using the standardized uptake value for quantification of FDG uptake of tumours. Eur J Nucl Med. 1998;25: 559-564. 
Shimada, H., Uchiyama, Y., \& Kakurai, S. Specific effects of balance and gait exercises on physical function among the frail elderly. Clin Rehabil. 2003;17: 472-479.

Shimada, H., Kimura, Y., Suzuki, T., et al. The use of positron emission tomography and [18F]fluorodeoxyglucose for functional imaging of muscular activity during exercise with a stride assistance system. IEEE Trans Neural Syst Rehabil Eng. 2007;15: 442-448.

Shimada, H., Suzuki, T., Kimura, Y., et al. Effects of an automated stride assistance system on walking parameters and muscular glucose metabolism in elderly adults. $\mathrm{Br} \mathrm{J}$ Sports Med. 2008;42: 922-9.

Shimada, H., Hirata, T., Kimura, Y., et al. Effects of a robotic walking exercise on walking performance in community-dwelling elderly adults. Geriatr Gerontol Int. 2009a;9: 372-381.

Shimada, H., Kimura, Y., Lord, S.R., et al. Comparison of regional lower limb glucose metabolism in older adults during walking. Scand J Med Sci Sports. 2009b, 19: 389397.

Shimada H, Sturnieks D, Endo Y, et al. Relationship between whole body oxygen consumption and skeletal muscle glucose metabolism during walking in older adults: FDG PET study. Aging Clin Exp Res. 2010.

Simonsick, E.M., Guralnik, J.M., Volpato, S., Balfour, J., \& Fried, L.P. Just get out the door! Importance of walking outside the home for maintaining mobility: findings from the women's health and aging study. J Am Geriatr Soc. 2005;53: 198-203.

Sokoloff, L., Reivich, M., Kennedy, C., et al. The [14C]deoxyglucose method for the measurement of local cerebral glucose utilization: theory, procedure, and normal values in the conscious and anesthetized albino rat. J Neurochem. 1977;28: 897-916.

Stevens, J.E., Stackhouse, S.K., Binder-Macleod, S.A., \& Snyder-Mackler, L. Are voluntary muscle activation deficits in older adults meaningful? Muscle Nerve. 2003;27: 99-101.

Tashiro, M., Fujimoto, T., Itoh, M., et al. 18F-FDG PET imaging of muscle activity in runners. J Nucl Med. 1999;40: 70-76.

Thelen, D.G., Schultz, A.B., Alexander, N.B., \& Ashton-Miller, J.A. Effects of age on rapid ankle torque development. J Gerontol A Biol Sci Med Sci. 1996;51: M226-232.

Van Hook, F.W., Demonbreun, D., \& Weiss, B.D. Ambulatory devices for chronic gait disorders in the elderly. Am Fam Physician. 2003;67: 1717-1724.

van Uffelen, J.G., Chinapaw, M.J., van Mechelen, W., \& Hopman-Rock, M. Walking or vitamin B for cognition in older adults with mild cognitive impairment? A randomised controlled trial. Br J Sports Med. 2008;42: 344-351.

Waters, R.L., Lunsford, B.R., Perry, J., \& Byrd, R. Energy-speed relationship of walking: Standard tables. J Orthop Res. 1988;6: 215-222.

Winter, D.A. (1991). The biomechanics and motor control of human gait: normal, elderly and pathological. University of Waterloo Press, Waterloo.

Winter, D.A., Patla, A.E., Frank, J.S., \& Walt, S.E. Biomechanical walking pattern changes in the fit and healthy elderly. Phys Ther. 1990;70: 340-347.

Young, D.R., Masaki, K.H., \& Curb, J.D. Associations of physical activity with performancebased and self-reported physical functioning in older men: The Honolulu Heart Program. J Am Geriatr Soc. 1995;43: 845-854. 


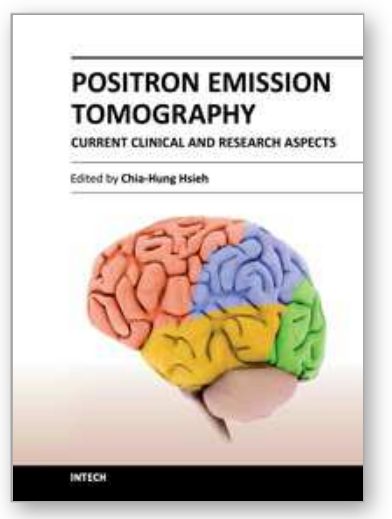

\author{
Positron Emission Tomography - Current Clinical and Research \\ Aspects \\ Edited by Dr. Chia-Hung Hsieh
}

ISBN 978-953-307-824-3

Hard cover, 336 pages

Publisher InTech

Published online 08, February, 2012

Published in print edition February, 2012

This book's stated purpose is to provide a discussion of the technical basis and clinical applications of positron emission tomography (PET), as well as their recent progress in nuclear medicine. It also summarizes current literature about research and clinical science in PET. The book is divided into two broad sections: basic science and clinical science. The basic science section examines PET imaging processing, kinetic modeling, free software, and radiopharmaceuticals. The clinical science section demonstrates various clinical applications and diagnoses. The text is intended not only for scientists, but also for all clinicians seeking recent information regarding PET.

\title{
How to reference
}

In order to correctly reference this scholarly work, feel free to copy and paste the following:

Hiroyuki Shimada (2012). Glucose Uptake During Exercise in Skeletal Muscles Evaluated By Positron Emission Tomography, Positron Emission Tomography - Current Clinical and Research Aspects, Dr. Chia-Hung Hsieh (Ed.), ISBN: 978-953-307-824-3, InTech, Available from: http://www.intechopen.com/books/positron-emissiontomography-current-clinical-and-research-aspects/glucose-uptake-during-exercise-in-skeletal-musclesevaluated-by-positron-emission-tomography

\section{INTECH}

open science | open minds

\section{InTech Europe}

University Campus STeP Ri

Slavka Krautzeka 83/A

51000 Rijeka, Croatia

Phone: +385 (51) 770447

Fax: +385 (51) 686166

www.intechopen.com

\section{InTech China}

Unit 405, Office Block, Hotel Equatorial Shanghai

No.65, Yan An Road (West), Shanghai, 200040, China

中国上海市延安西路65号上海国际贵都大饭店办公楼405单元

Phone: +86-21-62489820

Fax: +86-21-62489821 
(C) 2012 The Author(s). Licensee IntechOpen. This is an open access article distributed under the terms of the Creative Commons Attribution 3.0 License, which permits unrestricted use, distribution, and reproduction in any medium, provided the original work is properly cited. 